\title{
A Simple Preparation of Liquid Media for the Cultivation of Strict Anaerobes
}

Yoshihito Uchino* and Suzuki Ken-Ichiro

NITE Biological Resource Center (NBRC), National Institute of Technology and Evaluation; 2-5-8 Kazusakamatari, Kisarazu, Chiba 292-0818, Japan

\begin{abstract}
A simple and effective method for preparing pre-reduced liquid media for anaerobe cultivation is described and demonstrated using figures and movies. The usefulness of this method has been verified during professional practice in the NBRC culture collection.
\end{abstract}

Keywords: Culture media preparation; Pre-reduced liquid media; Strict anaerobes; Modified Hungate method

\section{Introduction}

Many researchers believe that handling strictly anaerobic microorganisms is difficult, and that specialized media, equipment, and techniques are required for their cultivation. Without a doubt, the preparation of liquid media for the growth of strict anaerobes may be difficult for beginners who follow instruction manuals, even if the instructions are excellent. To this effect, here, we describe the basics for preparing pre-reduced liquid media for anaerobes, and demonstrate some steps using pictures and movies in an easy-to-understand manner. Our method does not require an anaerobic chamber. Once the anaerobic conditions are replicated in the culture vessels, one can handle the vessels in air and use aerobic incubators. We use the method of sealing culture vessels to generate individual anaerobic chambers; this technique was first introduced by Hungate [1].

The basic steps for preparing liquid media suitable for anaerobes are as follows: 1) preparation of culture media, 2) purgation of oxygen from the media by bubbling with anoxic gas, 3) sterilization of the sealed vessels by autoclaving, and 4) addition of reducing agents. Strict anaerobes grow at a low redox potential, and this cannot be achieved by oxygen purging alone; thus, the reducing agents are added. Many manuals instruct dispensing the media into each culture vessel after oxygen purging or autoclaving; this, however, may cause oxygen exposure or contamination. In this method, we first dispense the media into the culture vessels, followed by flushing oxygen-free gas, sealing the vessels, and autoclaving. Autoclaving works twofold: it sterilizes the vessel and purges oxygen. The method is successfully applicable to highly oxygen-sensitive microorganisms, such as methanogens.

\section{Equipments}

\section{Culture vessels for anaerobic cultivation}

A few types of culture vessels for growth of strict anaerobes are available commercially. These vessels are tightly closed with butyl rubber septa secured in place by aluminum center hole caps ((or center tear-off caps Figure 1ab) or plastic screw hole caps). The butyl rubber is usually used as the stopper on anaerobic culture vessels owing to its very low permeability to gases. A flange-type stopper (Figure 1b) is suitable for efficient gas flushing as shown in Movie 1. Some tools for crimping and de-capping aluminum caps are available commercially (Figure 1c, 1d). Sterile needles and syringes are used to transfer materials from and to the vessel through the butyl rubber septa. Tuberculin syringes (1 $\mathrm{ml}$ ) fitted with disposable 27-gauge needles are most commonly used.

\section{Gas supply}

Nitrogen, argon, hydrogen, carbon dioxide, or mixtures thereof are often used for the cultivation of anaerobes. These gases are supplied in compressed gas cylinders and controlled by pressure regulators and flow valves (Figure 2a). If high-purity gases are used, the oxygen removal system (e.g., heated copper column) located between the regulator and the gassing nozzle need not be used (Figure 2b). A stainless steel nozzle $(25 \times 0.1 \mathrm{~cm})$ is used to directly inject anoxic gas into the medium in order to exclude dissolved oxygen (Figure 2c). Incidentally, we use the Culture System for Anaerobic Gas Jet Method (Sanshin Industrial, Yokohama, Japan) which contains the copper column, the stainless steel nozzle and so on, but it is not necessary to use this system; any system that meets the criteria mentioned above can be used.

It is important that one should be aware of and observe all the safety precautions associated with the use of the materials, tools, and other equipments.

\section{Media preparation}

Mix all the growth media ingredients, except heat-sensitive materials (e.g., vitamins), precipitate-causing materials (e.g., bicarbonate), and the reducing agents (e.g., sulfide and cysteine hydrochloride) in distilled water, adjust the $\mathrm{pH}$ to the desired level, and dispense this mixture into the culture vessels (at $10 \mathrm{ml}$ medium $/ 20$ $\mathrm{ml}$ vessel). If the $\mathrm{pH}$ is affected by autoclaving or by the subsequent addition of reagents, the $\mathrm{pH}$ should be adjusted later. Resazurin, a blue dye, is included in the media $(1 \mathrm{mg} / \mathrm{L})$ to monitor the redox potential of the media. On reduction, its color changes from blue to pink and from pink to colorless (Figures 2c, 6a, and 6b).

\section{Oxygen purging and sterilizing}

Regarding the kind of the gas used, follow the recipes of the media.

*Corresponding author: Dr. Yoshihito Uchino, NITE Biological Resource Cente (NBRC), National Institute of Technology and Evaluation; 2-5-8 Kazusakamatari, Kisarazu, Chiba 292-0818, Japan, Tel: +81-438-20-5763; Fax: +81-438-52-2329; E-mail: uchino-yoshihito@nite.go.jp

Received August 26, 2011; Accepted November 21, 2011; Published Novembe 23, 2011

Citation: Uchino Y, Ken-Ichiro S (2011) A Simple Preparation of Liquid Media for the Cultivation of Strict Anaerobes. J Pet Environ Biotechnol S3:001. doi:10.4172/2157-7463.S3-001

Copyright: ( 2011 Uchino Y, et al. This is an open-access article distributed unde the terms of the Creative Commons Attribution License, which permits unrestricted use, distribution, and reproduction in any medium, provided the original author and source are credited. 
Citation: Uchino Y, Ken-Ichiro S (2011) A Simple Preparation of Liquid Media for the Cultivation of Strict Anaerobes. J Pet Environ Biotechnol S3:001. doi:10.4172/2157-7463.S3-001

The purging processes must be carried out in a draft chamber for safety reasons. The medium within each vessel is purged by inserting the end of the gas nozzle into the liquid for 5-10 min. The oxygen-free gas is supplied moderately enough not to bubble over the vessel. The rubber septum is placed on top of the vessel during gassing, and then, the vessel is rapidly sealed with the septum while removing the nozzle, leaving no chance for oxygen to enter. The septa seal at the top of the vessel is tightened with aluminum or plastic caps (Movie 1).

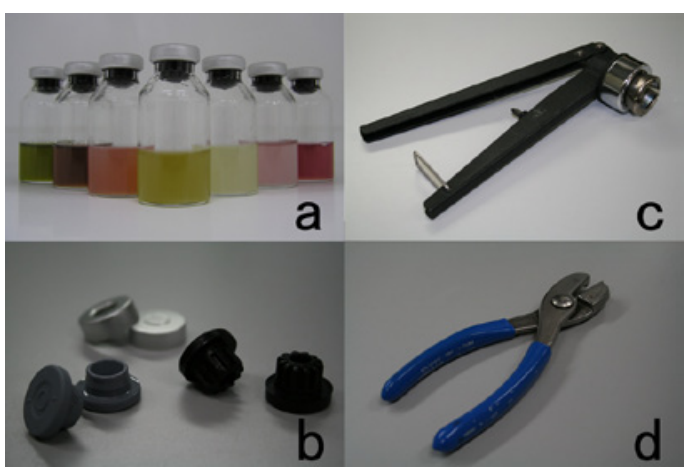

Figure 1: Culture vessels and tools for anaerobic cultivation. (a) The vessels culturing anoxygenic phototrophic bacteria; (b) Caps used for anaerobic cultivation. Butyl rubber septa (front right), teflon-coated butyl rubber septa (front left), and aluminum caps (back); (c) Hand crimper for crimping aluminum caps on vials, and (d) decapper for removing aluminum caps from the vials.

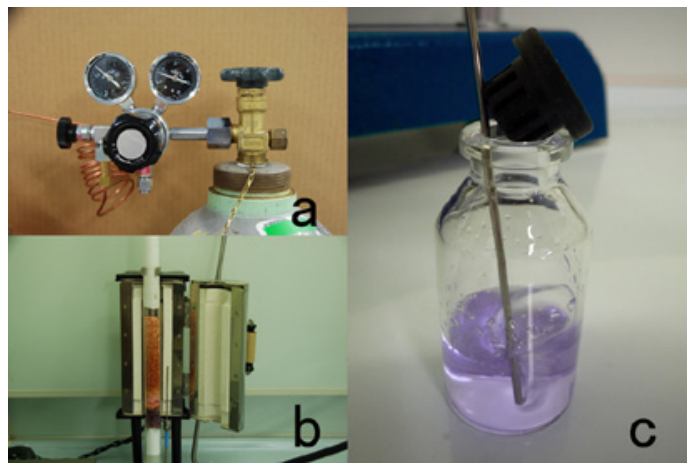

Figure 2: Gas supply equipments. (a) A gas regulator attached to a compressed gas cylinder, (b) Heated copper column, (c) Oxygen purging while putting the nozzle tip into the liquid directly.

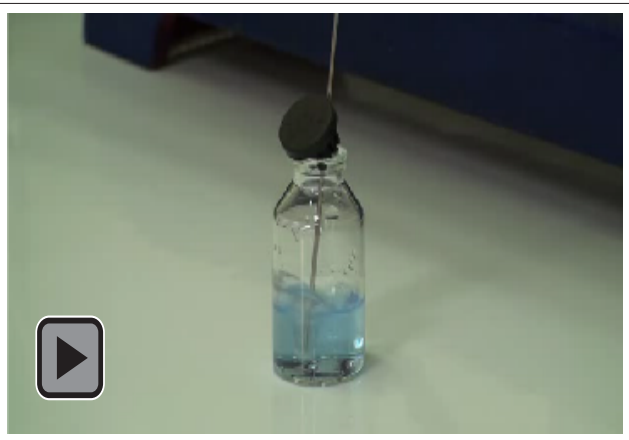

Movie 1: Oxygen purging with anoxic gas.

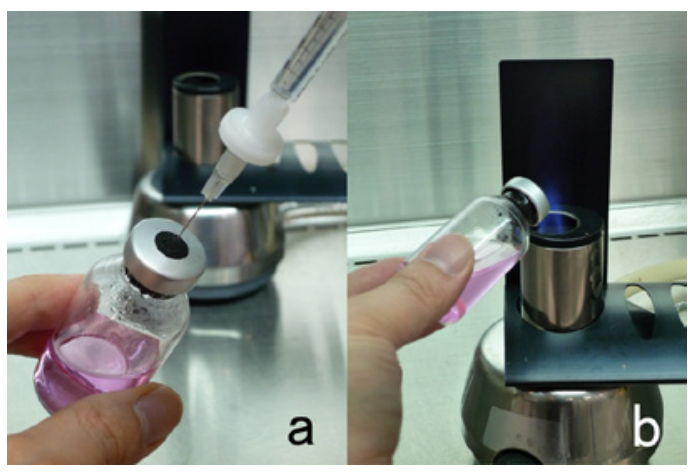

Figure 3: Aseptic techniques on a clean bench. Filter sterilization for heatsensitive materials, (b) Sterilization of the top of culture vessel by flaming.

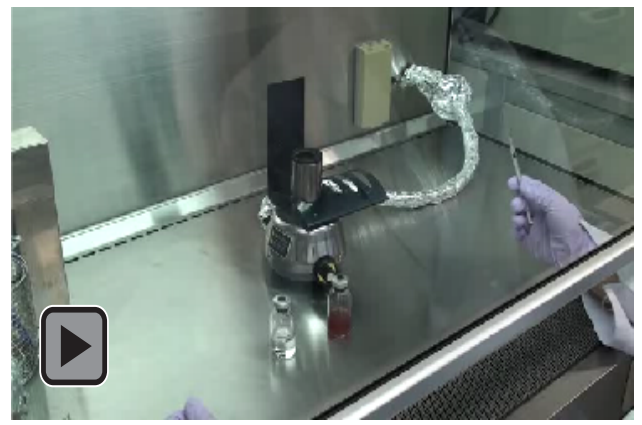

Movie 2: The bottle (right) contains culture of a phototrophic bacterium The supplement solution which could contain precipitate-causing materials, heat-sensitive materials or reducing agents is transferred in the same way.

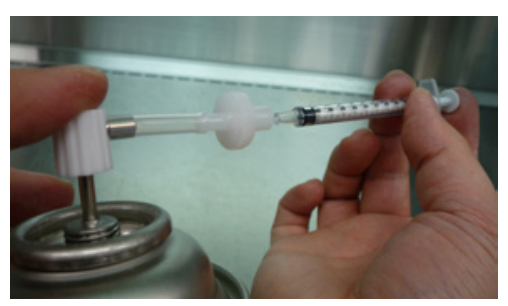

Figure 4: Flushing a syringe with nitrogen gas. Syringe is flushed 5 or more times with nitrogen gas to remove the air. After assembly with needle, nitrogen is ejected from the syringe through the needle.

Then, the sealed vessel is autoclaved at $121^{\circ} \mathrm{C}$ for $15 \mathrm{~min}$. The medium undergoes further oxygen purging during autoclaving.

\section{Addition of supplements and reducing agents}

After autoclaving, sterile and anoxic supplement solutions are injected into the medium. Prior to injection, the supplement solutions and reducing agents should be oxygen-purged with an anoxic gas and autoclaved for sterilization. Heat-sensitive materials (e.g., vitamins) should be subjected to filter sterilization (Figure 3a). Sterile needles and syringes are used to inject materials into the medium as mentioned earlier, and for all operations after autoclaving, aseptic and anaerobic handling is required. The top of the vessel should be sterilized by wiping with $70 \%$ ethanol, followed by flaming (Figure $3 \mathrm{~b}$ ); a clean bench should be used for sterilization. The anaerobic supplement 
Citation: Uchino Y, Ken-Ichiro S (2011) A Simple Preparation of Liquid Media for the Cultivation of Strict Anaerobes. J Pet Environ Biotechnol S3:001. doi:10.4172/2157-7463.S3-001

solutions are transferred using syringes as shown in Movie 2. During the transfer, the vessel should be placed upside down, the needle should be injected through the rubber septum, and the required volume should be withdrawn with the syringe plunger. By flicking the syringe

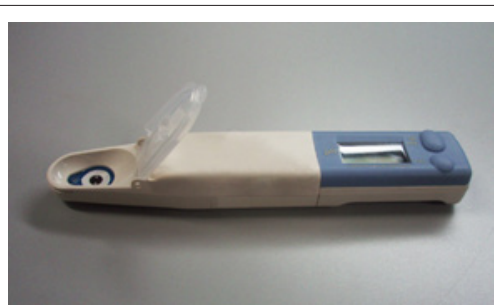

Figure 5: Compact $\mathrm{pH}$ meter. As for the medium sealed in a vial, the $\mathrm{pH}$ is measured using the compact $\mathrm{pH}$ meter in small volumes.

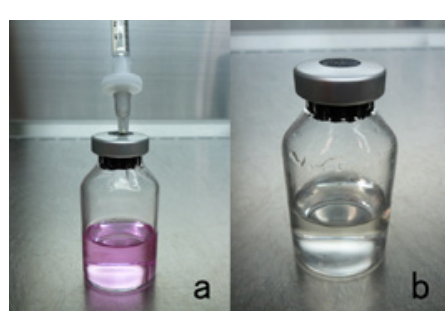

Figure 6: Color change of resazurin on addition of the reducing agent. (a) Oxygen-purged medium (pink) in which the reducing agent is being injecting, (b) Medium (colorless) after adding the reducing agent. with a finger, the bubbles trapped in the syringe can be expelled. We recommend that the dead space in the syringe be displaced by purging with filter-sterile nitrogen gas prior to transferring the anaerobic solution, as shown in Figure 4. The suitable volume injected out by 1 -ml tuberculin syringes is $100 \mu \mathrm{l}$, and the concentration of the reagents should be set accordingly. The desired $\mathrm{pH}$ should be adjusted using a compact $\mathrm{pH}$ meter (Figure 5), and by injecting sterile and anoxic $\mathrm{pH}$ adjusting agents with a syringe, without opening the vessel.

The reducing agents should be added last. The resazurin present in the medium turns colorless from pink on adding the reducing agents (Figure 6). All strict anaerobes require a minimum redox potential (Eh, $-110 \mathrm{mV}$ ) at which resazurin turns colorless; the colorlessness of the medium is a prerequisite. The reducing agents most commonly used are sodium sulfide, cysteine hydrochloride, thioglycolate (with ascorbate), dithiothreitol, dithionite, etc. The kind and quantity of the reducing agents to be added to medium are given in the recipes of the media. The NBRC online catalogue contains several recipes for anaerobic media along with links for information about each strain (http://www.nbrc. nite.go.jp/e/alphabet-e.html).

\section{Acknowledgement}

We thank the staff of NBRC culture collection, especially Ms. Shinobu Iwasak for her assistance.

\section{Reference}

1. Hungate RE (1969) A roll tube method for cultivation of strict anaerobes: Methods in Microbiology Academic Press, London, UK 3B:117-132. 